\title{
Electrophysiological Features of Neurons in the Mesencephalic Trigeminal Nuclei
}

\author{
Jun-Ling Xing ${ }^{a} \quad$ San-Jue $\mathrm{Hu}^{a} \quad$ Jing Yang ${ }^{b}$ \\ ${ }^{a}$ Institute of Neuroscience, The Fourth Military Medical University, and b The 323 Military \\ Hospital, Xi'an, PR China
}

\section{Key Words}

Mesencephalic trigeminal nuclei · Excitability classification · Transition · Subthreshold membrane potential oscillation · Resonance

\begin{abstract}
Mesencephalic trigeminal nucleus (Mes V) neurons represent an uncommon class of primary sensory neurons. Besides receiving somatosensory information, Mes $V$ neurons are also involved in regulating multisensory information. The present review first describes the passive features as well as three important currents, followed by a distinct excitability classification and a description of the excitability transition of Mes $V$ neurons. Furthermore, their resonance property, the existence of membrane oscillation and electrical coupling which may promote strong synchronization, as well as their function in controlling stretch reflex activity, are discussed.

(c) 2015 S. Karger AG, Basel
\end{abstract}

\section{Introduction}

Mesencephalic trigeminal nucleus (Mes V) neurons are traditionally regarded as sensory neurons correlated to the stretch reflex. In amphibians, other than receiving somatosensory information, Mes $\mathrm{V}$ neurons can also be driven synaptically through visual connection and thus may play an integrative role in regulating multisensory information [1]. The Mes $V$ has other roles including transferring information of vibrissa movements to other central nervous system (CNS) areas [2] as well as regulating feeding and exploratory behavior [3]. According to the morphological characteristics and terminating patterns, three types of neurons could be classified in Mes V: (1) jaw muscle spindle afferent Mes V neurons, (2) periodontal ligament mechanoreceptor afferent Mes V neurons and (3) interneurons [4, 5]. However, some investigators think that the small multipolar interneurons are not necessarily Mes $\mathrm{V}$ neurons.

The 323 Military Hospital

6 West Jian-she Road

Xi'an 710054 (PR China)

E-Mail yangjing323@163.com
Jun-Ling Xing

Institute of Neuroscience, The Fourth Military Medical University

17 Chang Le West Road

Xi'an 710032 (PR China)

E-Mail xingjunl@fmmu.edu.cn 
Rather, they might be other types of neurons from the surrounding areas [4]. As multipolar Mes V neurons may exhibit different properties from pseudounipolar ones, the electrophysiological features reviewed in this paper are mainly referring to common Mes V neurons which receive projections from muscle spindles in the jaw-closing muscles and periodontal mechanoreceptors [6]. Mes V neurons were identified on the basis of their morphological characteristics: a spherical or oval cell body, a large nucleus, a prominent single nucleolus and a pseudounipolar process [5]. The electrophysiological features, reflecting the input-output relation of a single cell, are important for exploring the basic function of a cell within a circuit. As unique primary sensory neurons in the CNS, the majority of Mes V neurons may have passive properties similar to those of other peripheral sensory neurons such as dorsal root ganglion (DRG) and trigeminal ganglion (TG) neurons.

Neurons are excitable, though cells with identical inputs may respond differently. It was Hodgkin who was the first to study crustacean nerve axons to distinguish neuronal excitability into three classes according to responses of the cell to applied stimuli [7]. Correspondingly, alterations in certain parameters, such as the dynamic characteristics of a current, can be crucial, since these change the neuron's firing behavior from one type to another [8-12].

Resonance is an inherent property that some neurons have to respond selectively to input signals at a proper frequency $[13,14]$. It occurs when there are complex interactions among different passive membrane properties which are time and voltage dependent [13]. Coherent network oscillations are correlated with different behavioral states in the brain and are determined by intrinsic resonance properties [13]. As parts of the intrinsic neuronal properties, resonance and membrane oscillation have been found both in the CNS [15-22] and in peripheral sensory neurons including Mes V, TG and DRG neurons [23, 24].

In this paper, we review findings primarily from passive electrophysiological features, following three important currents which play crucial roles in the neuronal behavioral variety of Mes V neurons. Subsequently, studies on the distinct excitability classification and excitability transition of Mes $\mathrm{V}$ neurons, combined with analyses of underlying ionic currents including the persistent sodium current $\left(I_{\mathrm{NaP}}\right)$ and the noninactivating 4-aminopyridine (4-AP)-sensitive potassium current $\left(I_{4-\mathrm{AP}}\right)$, are introduced. Furthermore, we will discuss their resonance property, the existence of membrane oscillation and electrical coupling which may promote strong synchronization as well as their function in controlling stretch reflex activity.

\section{The Membrane Properties of Mes V Neurons}

Most of the passive characteristics of adult Mes V neurons reported are similar, with a resting membrane potential (RMP) of $-56.4 \pm 6.5 \mathrm{mV}$ and an input resistance $\left(\mathrm{R}_{\text {in }}\right)$ of $143.6 \pm 52.9 \mathrm{M} \Omega$, no matter whether they come from whole-cell current-clamp recording of dissociated cells [4] or from brain slices [25]. Development has little effect on the RMP but a prominent effect on $\mathrm{R}_{\mathrm{in}}$, which is supported by work from Chandler and coworkers [26]. In their investigation, based on Sprague Dawley (SD) rats aged postnatal day (P)2-12 and using in vitro whole-cell patch-clamp recording, they found that the $\mathrm{R}_{\text {in }}$ decreased gradually from $225.8 \pm 166.8$ to $106.2 \pm 64.4 \mathrm{M} \Omega$, with the cell body having increased between P2 and P12. Correspondingly, the whole-cell capacitance gradually increased from $35.7 \pm 8.8$ to $62.8 \pm 12.4 \mathrm{pF}$. Similar results have been found by Yang et al. [27], who utilized 140 Mes V neurons from SD rats aged P9-14. Compared to the above data, there are some exceptional studies in which mean $R_{\text {in }}$ ranges from 13.4 to $24 \mathrm{M} \Omega$ are reported $[28,29]$. 
The neurons in Mes V seldom fire in the resting state; in fact, they respond by only one spike to a depolarizing step of short duration $(0.06 \mathrm{~ms})$ [4]. In the study by Yoshida and Oka [4], the amplitude of the action potential (AP) was $92.5 \pm 11.9 \mathrm{mV}$ and the spike duration was $0.94 \mathrm{~ms}$. Repetitive discharges could be evoked if the depolarizing current pulse was extended to above $350 \mathrm{~ms}$. The authors reported that the great majority of Mes V neurons responded to the stimuli with the maximum spike number, usually less than 3 , acting as a rapidly adapting neuron, while other neurons belonged to slowly adapting neurons which produced increasing spikes when the current intensity was increased.

In later investigations, rhythmical burst discharges became a remarkable subject. In this condition, cells discharge high-frequency trains of spikes in response to a depolarizing current $[26,30]$. The following properties of rhythmical burst discharges could be observed: the mean threshold for initiating rhythmical burst was $-51 \pm 4 \mathrm{mV}(-44$ to -60$)$, the range of mean cycle periods (0.4-19.0 s) and the burst durations (0.04-5.1 s) were quite different between neurons, and the mean intraburst firing frequency was $99 \pm 19 \mathrm{~Hz}(69-156)$ when measured at the threshold for rhythmical bursts [26]. Further study suggested that the subthreshold oscillation frequency determined the intraburst spike frequency and the high rate of discharge produced on Mes V neurons were actually caused by the underlying high-frequency subthreshold oscillation, a relation which will be introduced in more detail in the following section. Compared to the TG neurons which mediate the nociceptive sensation of the masseter muscle, Mes V neurons only have brief AP of a limited duration of $1 \mathrm{~ms}$ [31]. An interesting phenomenon is that there are two kinds of soma spikes in Mes V neurons, one initiating directly from the soma, the other caused by invasion of an axonal spike. This is true in immature animals - though in mature animals, it is difficult to record an antidromic discharge in Mes V somata by stimulating a jaw muscle nerve. Some researchers think that the impulses from the peripheral sensor (spindle or mechanoreceptor) run through the peripheral process directly across to the central axon at their joint point, so as not to collide away within the unipolar trunk.

\section{Three Important Currents in Mes V Neurons}

For Mes $\mathrm{V}$ neurons of the rapidly adapting type, the depolarization-induced AP was reversibly inhibited by tetrodotoxin (TTX) or by eliminating $\mathrm{Na}^{+}$from the bathing medium, suggesting a TTX-sensitive $\mathrm{Na}^{+}$channel foundation [29]. However, in conditions of various stimuli, many ion channels participate in the electrophysiological activity of MesV neurons, of which $I_{4-\mathrm{AP}}, I_{\mathrm{NaP}}$ and the hyperpolarization-activated cationic currents $\left(I_{\mathrm{h}}\right)$ are especially important.

\section{4-AP-Sensitive Potassium Current}

$I_{4-\mathrm{AP}}$ widely occurs in mammalian CNS [32-34], sensory neurons and trigeminal motor nuclei, among other locations $[35,36]$. It is now ascertained that there are two distinct 4-AP-sensitive outward currents: one is transient ( $I_{\text {TOC-S; }} I_{\text {Aslow }}$, slow inactivating component of the A type $\mathrm{K}^{+}$current), and the other is sustained (noninactivating-component $I_{4-\mathrm{AP}}$ ) [30]. $I_{4-\mathrm{AP}}$ (which is blocked by 30-100 $\mu \mathrm{M} 4-\mathrm{AP}$ ) is more sensitive than $I_{\mathrm{TOC}-\mathrm{S}}$ (blocked at 1-5 mM 4-AP). In Mes V neurons, under the application of $100 \mu \mathrm{M} 4-\mathrm{AP}, I_{4-\mathrm{AP}}$ was recorded [30]. Thus, in Mes $\mathrm{V}$ neurons, the 4-AP-sensitive current components of $I_{\mathrm{TOC}-\mathrm{S}}$ and $I_{4 \text {-AP }}$ determine the sustained period of spike trains. In these, the noninactivating $I_{4 \text {-AP }}$ plays a critical role in determining whether cells exhibit accommodation or sustained repetitive discharge. Moreover, it is now accepted that Mes V neurons, especially those from P12-18 immature neurons, have two kinds of soma spikes: one initiated directly from the soma, the other caused by invasion of an axonal spike. $I_{4-\mathrm{AP}}$ has been reported to have different effects on these two original 
spikes: facilitating axonal spike invasion and suppressing soma initiation [37]. Consistent with this, $\mathrm{K}_{\mathrm{v}} 1.1$ and $\mathrm{K}_{\mathrm{v}} 1.6$, which belong to the 4-AP-sensitive potassium family, were detected in the soma but not in the stem axon of Mes V neurons [37].

\section{Persistent Sodium Current}

$I_{\mathrm{NaP}}$, which is a voltage-dependent and noninactivating inward current when given a depolarized stimulus, has been extensively found in mammalian neurons located in striated (cardiac) muscles, skeletal muscles and the CNS [38-42]. By applying depolarizing voltage ramps from -90 to $10 \mathrm{mV}$ within $3 \mathrm{~s}$, Wu et al. [43] isolated $I_{\mathrm{NaP}}$ in Mes $\mathrm{V}$ neurons in neonatal SD rats. $I_{\mathrm{NaP}}$ was activated around $-76 \mathrm{mV}$, which is $20 \mathrm{mV}$ more negative than the value for the transient sodium current, and peaked at $-48 \mathrm{mV}$. This is similar to the properties in other neurons reported previously [44-46]. The peak amplitude of $I_{\mathrm{NaP}}$ in Mes $\mathrm{V}$ varies widely from cell to cell (ranging from -133.7 to $-918.7 \mathrm{pA}$ ), though the current density is between 4.6 and $6.9 \mathrm{pA} / \mathrm{pF}$. The inactivation of $I_{\mathrm{NaP}}$ is also time and voltage dependent, and the recovery after inactivation needs a few seconds ( $\tau_{\text {onset }}=2.04 \mathrm{~s}, \tau_{\text {recov }}=2.21 \mathrm{~s}$ ). Compared to TTX, a relatively special blocker for $I_{\mathrm{NaP}}$ was proposed to be riluzole. In Mes $\mathrm{V}$ neurons, riluzole reduced the peak $I_{\mathrm{NaP}}$ by $53 \%$ at $2 \mu \mathrm{M}$ and by $81 \%$ at $5 \mu \mathrm{M}$. Thus, a concentration of riluzole $<5 \mu \mathrm{M}$ could be considered a relatively special blocker compared to TTX in Mes V neurons [26].

As regards function, $I_{\mathrm{NaP}}$ is crucial for determining excitability behavior. Some evidence shows that $I_{\mathrm{NaP}}$ is the amplification factor of subthreshold membrane potential oscillation (SMPO) in many different neurons, and blockade of $I_{\mathrm{NaP}}$ could decrease SMPO or even abolish the burst discharge $[15,47-50]$. Apart from this, $I_{\mathrm{NaP}}$ is also the amplifier of many membrane resonance currents $[51,52]$ and exerts an amplified action on the subthreshold excitatory postsynaptic potential [53-55]. By dual recording at the soma and the axon hillock of Mes $\mathrm{V}$ neurons, Kang et al. [56] believe to have shown that $I_{\mathrm{NaP}}$ may be involved in spike initiation at the axon hillock, though $I_{\mathrm{NaP}}$ detected on the soma of Mes $\mathrm{V}$ neurons was considered to underlie the bursting behavior. In support of this, sodium currents (transient, resurgent and persistent) can be recorded in Mes V neurons, where $I_{\mathrm{NaP}}$ is considered to be the current flowing during the interspike interval of high-frequency firing, which, along with the transient sodium current flowing during the upstroke of AP and the resurgent sodium current flowing at the peak of afterhyperpolarization, contributes to Mes V electrogenesis [57]. The $\mathrm{Na}_{\mathrm{v}} 1.6$ isoform is partly responsible for the $I_{\mathrm{NaP}}$ composition; thus, in $\mathrm{Na}_{\mathrm{v}} 1.6^{-/-}$mice, a reduced impedance-frequency relationship and attenuated SMPO can be observed in Mes V neurons compared to wild-type littermates [58]. Moreover, in Mes V neurons, the generation of SMPO and resonance are related to both $I_{\mathrm{NaP}}$ and $I_{4-\mathrm{AP}} . I_{\mathrm{NaP}}$ could be reversely regulated by 5 -HT through a cAMP/PKA pathway [59].

\section{Hyperpolarization-Activated Cationic Currents}

Hyperpolarization-activated cyclic nucleotide-gated (HCN) channels are intermembrane proteins that serve as nonselective ligand-gated cation channels in a variety of cells. HCN channels are encoded by four genes (HCN1, 2, 3 and 4) and are widely expressed throughout the heart and the CNS $[60,61]$. The current mediated by HCN (hyperpolarization-activated cationic inward current, $I_{\mathrm{h}}$ ), along with $I_{\mathrm{KIR}}$, is a typical anomalous inward rectification current which is slowly activated by hyperpolarization to generate an inward current. $I_{\mathrm{h}}$ is permeable to both $\mathrm{Na}^{+}$and $\mathrm{K}^{+}$and can be activated by $-60-$ to $-90-\mathrm{mV}$ hyperpolarizing square-wave stimulation $[62,63]$. It can be blocked by extracellular $\mathrm{Cs}^{+}$at a low concentration, though ZD7288 is the specific inhibitor for $I_{\mathrm{h}}[25,64]$.

In 1998, Khakh and Henderson [65] first recorded $I_{\mathrm{h}}$ in Mes $V$ neurons and found that membrane hyperpolarization (from -62 to $-132 \mathrm{mV}$ ) can activate $I_{\mathrm{h}}$ with a half-maximal activation potential $\left(\mathrm{V}_{1 / 2}\right)$ of $-93 \mathrm{mV}$, maximal currents $\left(I_{\max }\right)$ of around $1 \mathrm{nA}$ and a reversal potential 
of $-57 \mathrm{mV}$. Using a hyperpolarization step protocol of 2-5 s, Tanaka et al. [25] detected the characteristics of the activation and deactivation dynamics of $I_{\mathrm{h}}$ in Mes V neurons, indicating that they could be described by two time constants. $I_{\mathrm{h}}$ has been found to be involved in a number of neuronal functions, participating in pacemaker activity in both the heart and the brain [66], setting an RMP which is self-limiting and stabilizing $[67,68]$, controlling membrane resistance and dendritic integration and regulating synaptic transmission [64]. Most of the abovementioned functions have been proved to exist in Mes V neurons, since blockade of $I_{\mathrm{h}}$ produces membrane hyperpolarization, extends the duration of afterhyperpolarization and decreases the firing frequency in the majority of Mes $\mathrm{V}$ neurons [25]. The function of mediating the lowfrequency membrane resonance of $I_{\mathrm{h}}$ has also been explored in Mes $\mathrm{V}$ neurons. There are studies reporting that $I_{\mathrm{h}}$ could be regulated by a subset of G protein-coupled receptors such as $\mathrm{P}_{2} \mathrm{Y}_{1}$ receptor [69]. In addition, activation of the $\mathrm{P} 2 \mathrm{X}$ receptor has been shown to have inhibiting effects on $I_{\mathrm{h}}$ [65]. $I_{\mathrm{NaP}}$ can be modulated not only by neurotransmitters but also by other ionic currents. For instance, activation of $I_{\mathrm{h}}$ results in the generation of an $\mathrm{Na}-\mathrm{K}$ pump current, which, in reverse, can facilitate the activation of slow $I_{\mathrm{h}}$ [70]. Mes $V$ neurons are covered by numerous microvilli, which express both h-channels and $\mathrm{Na}-\mathrm{K}$ pumps. $\mathrm{An}^{\mathrm{Na}}{ }^{+}$microdomain, which can be formed in such microvilli, underlies this bidirectional interaction [70].

\section{Neuronal Excitability Classification and Excitability Transition in Mes V Neurons}

Fundamentally, neuronal excitability, which can be divided into three classes according to responses of the cell to applied stimuli, was first put forward by Hodgkin [7]. Interneurons within the Mes $V$ area have been reported to be categorized into three subtypes: tonic-firing (type I), bursting-firing (type II) and spike-adaptive (type III) neurons [71]. On account of this, after a series of studies, Yang et al. [27] showed that Mes V neurons in rats can be classified into three types. As shown in figure 1A, class 1 neurons are sensitive to the strength of the stimuli applied (fig. 1A1). Dissimilarly, those of class 2 show a relatively stable firing frequency responding to the injected current (fig. 1A2). For class 3 excitability, usually a single AP is generated in response to current steps with different intensities, with 3-5 spikes generated even with extreme current stimuli (fig. 1A3). Of these three types of neurons, class 2 neurons, which are similar to the rhythmical burst neurons mentioned above, have received excessive attention $[26,28,30]$. With the help of nonlinear dynamics theory, these three classifications are described to have different dynamic bifurcation mechanisms [72].

Fig. 1. Excitability classification and transition in Mes V neurons. A1 Class 1 neurons respond to intracellular current steps with repetitive AP firing (left), which is current intensity dependent. This is shown in either the ramp stimulation-evoked response (middle) or in the F-I curve (right), which is approximately linear with respect to the current intensities. A2 Class 2 neurons respond to current stimuli with a constant spike frequency (left) which is current intensity independent, as shown in either the ramp stimulationevoked response (middle) or in the F-I curve (right). Inset There are SMPO along with the ramp currentevoked rhythmical bursting. A3 Class 3 neurons have a higher current intensity, which is required to evoke spikes, and the cell does not fire more than 3 spikes at the highest stimulus intensity (1,000 pA, left). A strong current ramp (1.5 nA) failed to evoke any response (right). B1 Excitability transition between class 2 and class 1 . The application of 4-AP transformed class 2 (left) to class 1 firing (middle); the addition of riluzole reversed the spike firing pattern back to class 2 firing, though at a lower frequency (right). B2 Excitability transition between class 2 and class 3. The application of riluzole transformed class 2 (left) to class 3 firing (middle); the addition of 4-AP reversed the spike firing pattern back to class 2 firing, though at a lower frequency (right). Reproduced from Yang et al. [27] with permission. ACSF = Artificial cerebrospinal fluid.

(For figure see next page.) 
A1

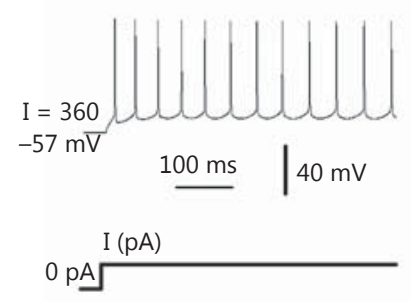

A2

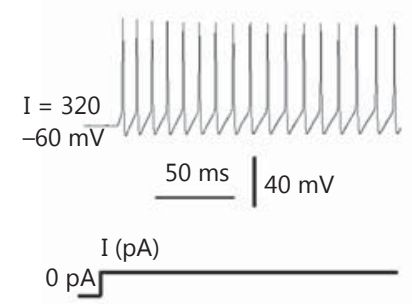

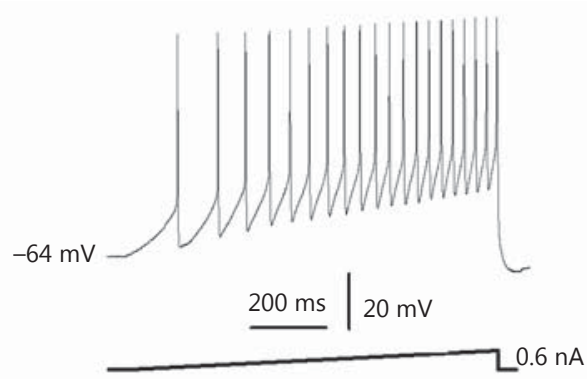
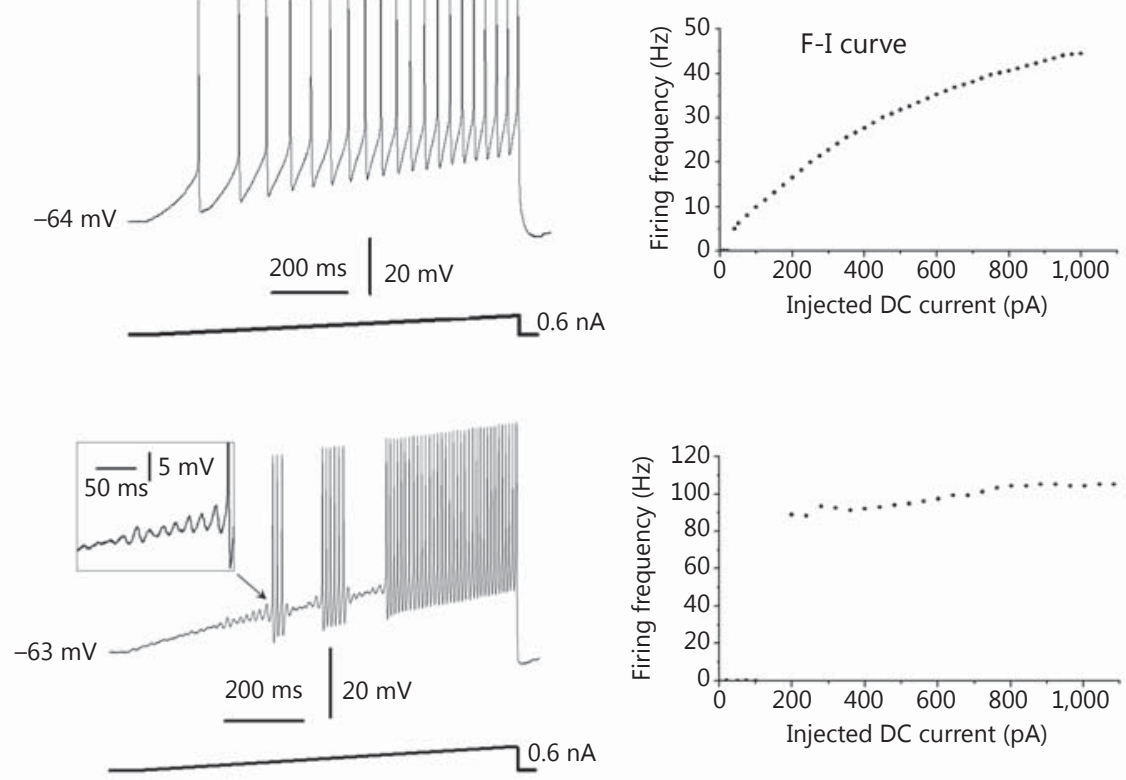

A3
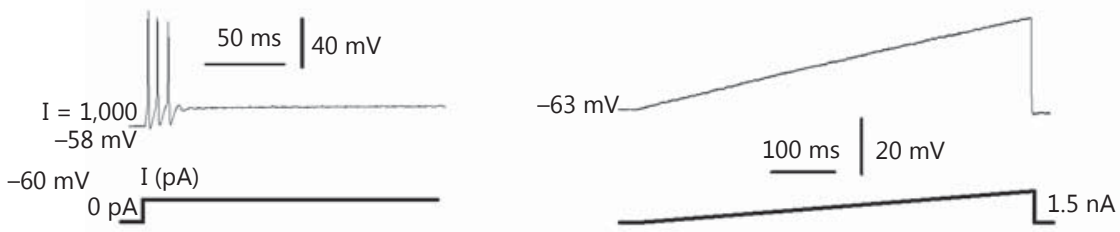

B1
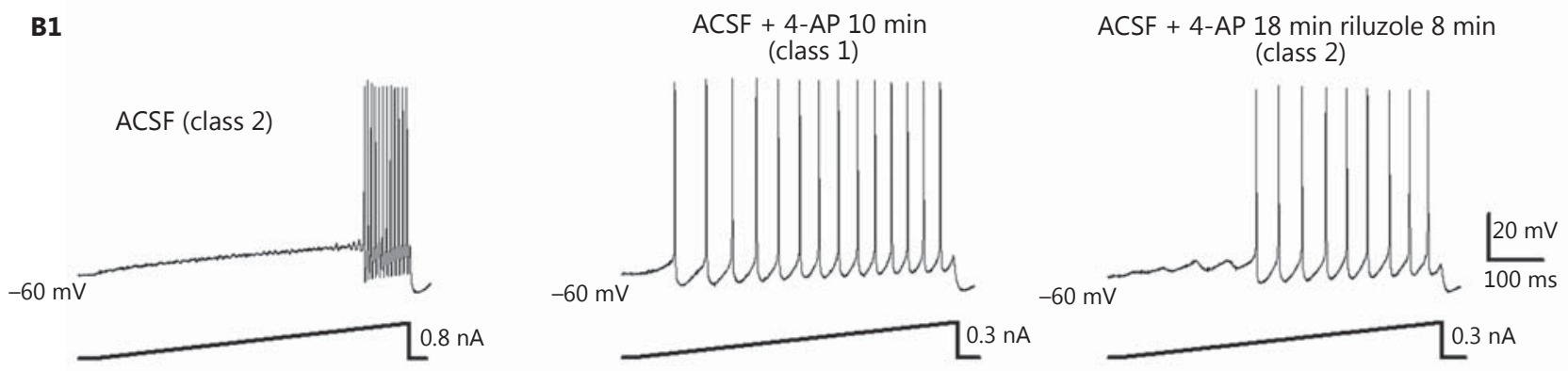

B2
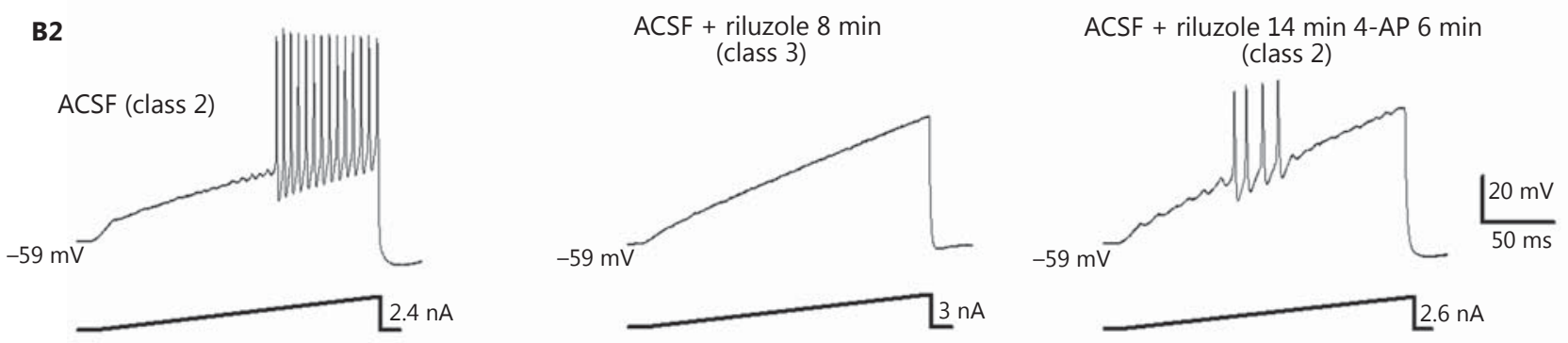
Ample evidence indicates that the discharge pattern of one type of neuron can be changed into another type under physiological conditions or an experimentally applied approach [8], which means that the input-output relation changes thereafter. In an interesting study on the chronic compression of rat sciatic nerves, it was shown that at a bifurcation point of critical sensitivity, patterns of bursting in an experimental pacemaker can be altered when the extracellular calcium concentration is changed $[12,73,74]$. For Mes V neurons, a theoretical study using mathematical models has demonstrated that small changes in the parameters related to ionic currents could lead to transitions between different classes of membrane excitability [72]. Different dynamic properties of ionic currents which were detected in different types of Mes $\mathrm{V}$ neurons provide experimental support for this possibility. Using a method similar to that of Del Negro and Chandler [30], Yang et al. [27] found that in class 2 Mes V neurons, $I_{4-\mathrm{AP}}$ was activated at a relatively depolarizing potential $(-57.5 \pm 5.9 \mathrm{mV})$, and $\mathrm{V}_{1 / 2}$ was $-48.0 \mathrm{mV}$. In class 3 neurons, the value was significantly different: the activation threshold of $I_{4-\mathrm{AP}}$ was about $-63.6 \pm 3.7 \mathrm{mV}$, and $V_{1 / 2}$ was $-54.2 \mathrm{mV}$. As to $I_{\mathrm{NaP}}$, the authors found that it was activated at about $-70 \mathrm{mV}$ in both class 2 and 3 neurons, with $\mathrm{V}_{1 / 2}$ at $-51.0 \mathrm{mV}(\mathrm{k}=-5.4)$ for class 2 and $-49.9 \mathrm{mV}(\mathrm{k}=-5.7)$ for class 3. Moreover, it was also found that the application of ZD7288 could increase the firing threshold of class 2 neurons, but the excitability of this cell type was unchanged [27].

A corresponding experiment has also been conducted to determine this excitability transition. It was found that reducing $I_{4-\mathrm{AP}}$ transformed class 3 to class 2 and finally to class 1 neurons, while suppressing $I_{\mathrm{NaP}}$ transformed class 2 to class 3 neurons [27]. More importantly, the application of 4-AP reversed the firing patterns that were abolished by riluzole (fig. 1B2), and, in turn, riluzole could reverse the excitation effect of 4-AP (fig. 1B1). These results suggest that electrophysiological behaviors (including neuronal excitability) are determined not only by the absolute magnitudes and inherent features of the critical currents (in this study, the critical currents were $I_{4-\mathrm{AP}}$ and $I_{\mathrm{NaP}}$ ) but also by the balance between these currents, which has been confirmed in another report [75]. In agreement with this standpoint, within a short period during the process of drug application, the neuronal behavior displays the mixed characteristics of both class 1 and class 2 . This strongly supports the abovementioned opinion that during the process of transformation, the relative proportion of $I_{4-\mathrm{AP}}$ and $I_{\mathrm{NaP}}$ keeps changing, which induces Mes V neurons to exhibit different excitability behaviors until a new balance is established.

\section{The SMPO of Mes V Neurons}

In the CNS, SMPO (i.e. membrane potential alternative fluctuation, usually expressed in millivolts) [76] is a common and important rhythmic activity. For a long time, there have been two opinions regarding the production of this rhythmicity. One opinion presumes that the synchronized rhythm stems from the neuronal network and that the neurons do not exert individual oscillation behaviors. The other presumes that the network rhythm comes from the intrinsic oscillation of each neuron within this area. More and more evidence for the existence of SMPO with different properties has been found in many encephalic regions of the CNS [77-81]. For example, theta wave frequencies $(2-7 \mathrm{~Hz})$ in hippocampal CA1 pyramidal neurons were generated by the subthreshold activity of three noninactivating currents [21, 79], SMPO in guinea pig cortical neurons is related to the potassium current and $I_{\mathrm{h}}[15,82]$, and SMPO in the nucleus olivaris inferior is calcium dependent [83]. In addition, SMPO at a high frequency can be observed in DRG, as in Mes V [84], which belongs to the primary afferent sensory neurons $[85,86]$.

In Mes V neurons, the work by Pedroarena et al. [28] was focused on a specific electrophysiological property, i.e. the ability to exhibit robust and sustained SMPO activity on 
membrane depolarization. They found that $52.6 \%$ of neurons generate periodic fluctuation with an amplitude of $2-4.3 \mathrm{mV}$ and a frequency of $108.9 \pm 15.5 \mathrm{~Hz}$ when the membrane potential is positive to $-53 \pm 2.3 \mathrm{mV}$. The following study by Wu et al. [26] investigated the change of SMPO during development. The oscillation amplitude and frequency had increased before P6 and further increased to a plateau level after P7-12. They also found that the amplitude of SMPO increased with further membrane depolarization, and AP were generated when the amplitude of SMPO reached the threshold. Along with the membrane potential depolarization, the peak frequency of SMPO switched to higher values until a maximum peak was reached, after which a decline occurred with further depolarization. The dominant frequency could be found after analyzing the power spectrum, which indicates that Mes $\mathrm{V}$ neurons have the tendency to respond selectively to a proper input frequency. Yang et al. [27] also found that about 39.3\% of Mes V neurons from P9-14 SD rats have an SMPO similar to that mentioned above - although it is even more interesting that the neurons which have an SMPO usually are classified as class 2 neurons, in which SMPO and, as a result, a constant firing frequency are observed. Otherwise, a persistent depolarizing stimulus could not evoke continued discharge from neurons in which SMPO does not occur. Thus, it is clear that even though SMPO is a phenomenon of low amplitude, it has a critical role in determining the neurons' input and output characteristics and may decide the final output state $[26,87]$. On the other hand, this oscillatory activity of Mes $\mathrm{V}$ neurons may play a very important role in jaw movement control, as originally postulated [28].

\section{The Resonance Phenomenon of Mes V Neurons}

SMPO could be considered the consequence of neural resonance. Resonance generally exists in nature; it has originally been regarded as a physical term defined as the tendency of a system to oscillate with greater amplitude at some frequencies than at others. Resonant frequencies are known as those frequencies at which the response amplitude is a relative maximum. In the nervous system, resonance indicates that it can distinguish input signals from their receiving frequency, so that the largest responses may be evoked by oscillatory inputs near the resonant frequency. Many neurons have this frequency selectivity (frequency preference), and they show the best response to an input stimulus in a very narrow frequency spectrum [21, 26, 82]. Exciting investigations show that resonance plays a key role in learning and memory as well as in synaptic plasticity when the brain neural network is in a coordinated condition $[21,25,26,78]$. Neurons possess resonance properties because of the interaction between their passive and active characteristics. The passive characteristics include two aspects: the membrane resistance formed by membrane permeability and the membrane capacitance formed by the membrane lipid bilayer, which form the low-pass filter in physical circuits and have the function of weakening the high-frequency components from the input signal. However, the membrane resistance-capacitance circuit is not necessarily the low-pass filter. As we know that, the TASK1/3 channel, which belongs to the two-pore domain channel family, has been widely accepted to be underlying the cell membrane potential and $\mathrm{R}_{\mathrm{in}}$. Various agents including volatile anesthetics and neurotransmitters can modulate the function of the TASK channel and thus can modulate the low-pass filter. The active elements of neurons are composed of voltage-gated ion channels [13] and have the function of inductors in the band-pass filter circuit, which permits passage of the high-frequency component of an input signal $[15,82]$. Only in this way, neurons can make a strong response to an input signal which has a close relationship to their own resonant frequency. 
Based on rats aged P2-12, Wu et al. [26] found that when holding the membrane potential at $-57 \mathrm{mV}$, Mes $\mathrm{V}$ neurons showed a voltage-dependent high-frequency resonance $(110 \mathrm{~Hz})$ in response to injection of a computer-generated impedance amplitude profile (swept-sine wave) input current of changing frequencies between 0 and $250 \mathrm{~Hz}$ into neurons. It was also clarified that the resonant current was $I_{4-\mathrm{AP}}$, and $I_{\mathrm{NaP}}$ could amplify this resonance. In a further investigation into class 2 neurons and part of class 3 neurons, we found another low-frequency resonance $(<10 \mathrm{~Hz})$ when holding the membrane potential at the hyperpolarization level (about-70 mV; data to be published). $50 \mu \mathrm{M}$ of 4-AP could abolish the high-frequency resonant peak but caused little or no change to the low-frequency resonant peak, while $10 \mu \mathrm{M}$ ZD7288 could abolish the low-frequency resonant peak but caused little or no change to the highfrequency resonant peak.

Neurons live in an environment which is full of noise, such as from stochastic opening and closure of the membrane ion channels and stochastic release of the neurotransmitters at synapses. It has recently been shown that neurons could take advantage of noise in detecting weak signals through stochastic resonance or autonomous stochastic resonance [88-91]. In our work, through observing and comparing effects of noise on the three classes of excitability, we found that only in class 2 neurons could a certain intensity noise reduce the membrane potential level at which SMPO is produced. The degree of such a reduction increases with the increment in noise intensity. This effect, along with the reduction of the spike threshold, contributes to increasing the excitability of class 2 neurons (data not shown). Indeed, the ion channel mechanism underlying this phenomenon still needs further investigation.

\section{Electrical Coupling Promotes Strong Synchronization}

The Mes V nucleus, formed by the somatic primary afferents initiating from jaw-closing muscles, is one of the first proofs supporting the existence of electrical synapses in the mammalian CNS. Four decades ago, classic experiments were carried out to interpret the 'short-latency depolarization' happening in Mes V, which suggested 'electrotonic coupling' as the mechanism for that phenomenon [92]. Lately, some proof that Mes V neurons form coupled networks via electrical and excitatory connections was reported by virtue of transmission electron microscopy [93] and horseradish peroxidase injection into masseter and temporalis muscles $[93,94]$. However, this direct approach for the detailed analyses of the features of these electrical contacts has not been further pursued until recently. Curti et al. [95] found that electrical coupling is restricted to small clusters of Mes V neurons. They also indicated that connexin-36-containing gap junctions mediated electrical transmission between Mes V neurons [96]. A kind of developmentally regulated brain protein, drebrin, was found to be located at axosomatic synapses and neuronal gap junctions; thus, it is an ideal marker of Mes V neurons [97]. Interestingly, the development of a coupling increase is responsible for the repetitive firing in these neurons, which parallels the development of inherent membrane properties. For instance, bursting is initiated after P6 and is largely determined by the oscillation frequency, which again emerges from the resonant properties of Mes $\mathrm{V}$ neurons [26]. Therefore, certain membrane properties may collaboratively interact with electric coupling to promote synchronization between Mes V neurons [95], but how these features contribute to their function, e.g. regulation of the stretch reflex, still needs further investigation. Moreover, since there are only few reports, more modeling work for the reproduction of electrophysiological behavior (such as excitability, resonance and SMPO as well as coupling activities) is needed, which could be a guide for introducing the functional state of MesV neurons [98]. 


\section{Conclusion}

The variety in excitatory responses of Mes $\mathrm{V}$ neurons does not only depend on the dynamic state of RMP such as SMPO and the frequency range of resonance, but it also rests on the classification of excitability and the underlying ionic currents. Whether the classification of excitability and its transition are also important for the variety in excitatory responses in other neurons is still unclear and will form an interesting topic for future research.

\section{Acknowledgements}

This work was supported by the National Natural Science Foundation of China (No. 31271185 and 81470060) and a grant from the 323rd Military Hospital (No. 2012323A02).

\section{References}

1 Pratt KG, Aizenman CD: Multisensory integration in mesencephalic trigeminal neurons in Xenopus tadpoles. J Neurophysiol 2009;102:399-412.

-2 Mameli O, Stanzani S, Russo A, Pellitteri R, Manca P, De Riu PL, Caria MA: Involvement of trigeminal mesencephalic nucleus in kinetic encoding of whisker movements. Brain Res Bull 2014;102:37-45.

-3 Yokoyama S, Kinoshita K, Muroi Y, Ishii T: The effects of bilateral lesions of the mesencephalic trigeminal sensory nucleus on nocturnal feeding and related behaviors in mice. Life Sci 2013;93:681-686.

4 Yoshida S, Oka H: Membrane properties of dissociated trigeminal mesencephalic neurons of the adult rat. Neurosci Res 1998;30:227-234.

5 Luo PF, Wang BR, Peng ZZ, Li JS: Morphological characteristics and terminating patterns of masseteric neurons of the mesencephalic trigeminal nucleus in the rat: an intracellular horseradish peroxidase labeling study. J Comp Neurol 1991;303:286-299.

6 Byers MR, O'Connor TA, Martin RF, Dong WK: Mesencephalic trigeminal sensory neurons of cat: axon pathways and structure of mechanoreceptive endings in periodontal ligament. J Comp Neurol 1986;250:181-191.

7 Hodgkin AL: The local electric changes associated with repetitive action in a non-medullated axon. J Physiol 1948;107:165-181.

8 Heyward P, Ennis M, Keller A, Shipley MT: Membrane bistability in olfactory bulb mitral cells. J Neurosci 2001; 21:5311-5320.

-9 Izhikevich EM: Solving the distal reward problem through linkage of STDP and dopamine signaling. Cereb Cortex 2007; 17:2443-2452.

10 Torkkeli PH, Sekizawa S, French AS: Inactivation of voltage-activated $\mathrm{Na}^{+}$currents contributes to different adaptation properties of paired mechanosensory neurons. J Neurophysiol 2001;85:1595-1602.

-11 Steriade M, Timofeev I, Durmuller N, Grenier F: Dynamic properties of corticothalamic neurons and local cortical interneurons generating fast rhythmic (30-40 Hz) spike bursts. J Neurophysiol 1998;79:483-490.

12 Yang J, Duan YB, Xing JL, Zhu JL, Duan JH, Hu SJ: Responsiveness of a neural pacemaker near the bifurcation point. Neurosci Lett 2006;392:105-109.

$\checkmark 13$ Hutcheon B, Yarom Y: Resonance, oscillation and the intrinsic frequency preferences of neurons. Trends Neurosci 2000;23:216-222.

14 Wang WT, Wan YH, Zhu JL, Lei GS, Wang YY, Zhang P, Hu SJ: Theta-frequency membrane resonance and its ionic mechanisms in rat subicular pyramidal neurons. Neuroscience 2006;140:45-55.

15 Gutfreund Y, Yarom Y, Segev I: Subthreshold oscillations and resonant frequency in guinea-pig cortical neurons: physiology and modelling. J Physiol 1995;483:621-640.

16 Hutcheon B, Miura RM, Puil E: Subthreshold membrane resonance in neocortical neurons. J Neurophysiol 1996;76:683-697.

17 Puil E, Meiri H, Yarom Y: Resonant behavior and frequency preferences of thalamic neurons. J Neurophysiol 1994;71:575-582.

18 Bertram R, Butte MJ, Kiemel T, Sherman A: Topological and phenomenological classification of bursting oscillations. Bull Math Biol 1995;57:413-439.

19 Leung LS, Yu HW: Theta-frequency resonance in hippocampal CA1 neurons in vitro demonstrated by sinusoidal current injection. J Neurophysiol 1998;79:1592-1596.

20 Pike FG, Goddard RS, Suckling JM, Ganter P, Kasthuri N, Paulsen O: Distinct frequency preferences of different types of rat hippocampal neurones in response to oscillatory input currents. J Physiol 2000;529:205-213.

$21 \mathrm{Hu}$ H, Vervaeke K, Storm JF: Two forms of electrical resonance at theta frequencies, generated by M-current, h-current and persistent $\mathrm{Na}^{+}$current in rat hippocampal pyramidal cells. J Physiol 2002;545:783-805. 
22 Haas JS, White JA: Frequency selectivity of layer II stellate cells in the medial entorhinal cortex. J Neurophysiol 2002;88:2422-2429.

23 Puil E, Gimbarzevsky B, Miura RM: Quantification of membrane properties of trigeminal root ganglion neurons in guinea pigs. J Neurophysiol 1986;55:995-1016.

24 Puil E, Gimbarzevsky B, Spigelman I: Primary involvement of $\mathrm{K}^{+}$conductance in membrane resonance of trigeminal root ganglion neurons. J Neurophysiol 1988;59:77-89.

25 Tanaka S, Wu N, Hsaio CF, Turman J Jr, Chandler SH: Development of inward rectification and control of membrane excitability in mesencephalic v neurons. J Neurophysiol 2003;89:1288-1298.

$26 \mathrm{Wu}$ N, Hsiao CF, Chandler SH: Membrane resonance and subthreshold membrane oscillations in mesencephalic V neurons: participants in burst generation. J Neurosci 2001;21:3729-3739.

-27 Yang J, Xing JL, Wu NP, Liu YH, Zhang CZ, Kuang F, Han VZ, Hu SJ: Membrane current-based mechanisms for excitability transitions in neurons of the rat mesencephalic trigeminal nuclei. Neuroscience 2009;163:799-810.

28 Pedroarena CM, Pose IE, Yamuy J, Chase MH, Morales FR: Oscillatory membrane potential activity in the soma of a primary afferent neuron. J Neurophysiol 1999;82:1465-1476.

29 Yamuy J, Pose I, Pedroarena C, Morales FR, Chase MH: Neurotrophin-induced rapid enhancement of membrane potential oscillations in mesencephalic trigeminal neurons. Neuroscience 2000;95:1089-1100.

-30 Del Negro CA, Chandler SH: Physiological and theoretical analysis of $\mathrm{K}^{+}$currents controlling discharge in neonatal rat mesencephalic trigeminal neurons. J Neurophysiol 1997;77:537-553.

-31 Connor M, Naves LA, McCleskey EW: Contrasting phenotypes of putative proprioceptive and nociceptive trigeminal neurons innervating jaw muscle in rat. Mol Pain 2005; 1:31.

-32 Klee R, Ficker E, Heinemann U: Comparison of voltage-dependent potassium currents in rat pyramidal neurons acutely isolated from hippocampal regions CA1 and CA3. J Neurophysiol 1995;74:1982-1995.

-33 Banks MI, Haberly LB, Jackson MB: Layer-specific properties of the transient K current (IA) in piriform cortex. J Neurosci 1996;16:3862-3876.

34 Johnston D, Christie BR, Frick A, Gray R, Hoffman DA, Schexnayder LK, Watanabe S, Yuan LL: Active dendrites, potassium channels and synaptic plasticity. Philos Trans R Soc Lond B Biol Sci 2003;358:667-674.

-35 Hsiao CF, Chandler SH: Characteristics of a fast transient outward current in guinea pig trigeminal motoneurons. Brain Res 1995;695:217-226.

-36 Stansfeld CE, Marsh SJ, Halliwell JV, Brown DA: 4-Aminopyridine and dendrotoxin induce repetitive firing in rat visceral sensory neurones by blocking a slowly inactivating outward current. Neurosci Lett 1986;64:299-304.

37 Saito M, Murai Y, Sato H, Bae YC, Akaike T, Takada M, Kang Y: Two opposing roles of 4-AP-sensitive $\mathrm{K}^{+}$current in initiation and invasion of spikes in rat mesencephalic trigeminal neurons. J Neurophysiol 2006;96:18871901.

-38 Tang Q, Ma J, Zhang P, Wan W, Kong L, Wu L: Persistent sodium current and $\mathrm{Na}^{+} / \mathrm{H}^{+}$exchange contributes to the augmentation of the reverse $\mathrm{Na}^{+} / \mathrm{Ca}^{2+}$ exchange during hypoxia or acute ischemia in ventricular myocytes. Pflugers Arch 2012;463:513-522.

-39 El-Bizri N, Kahlig KM, Shyrock JC, George AL Jr, Belardinelli L, Rajamani S: Ranolazine block of human $\mathrm{Na}_{\mathrm{v}} 1.4$ sodium channels and paramyotonia congenita mutants. Channels (Austin) 2011;5:161-172.

40 Crill WE: Persistent sodium current in mammalian central neurons. Annu Rev Physiol 1996;58:349-362.

-41 Yue C, Remy S, Su H, Beck H, Yaari Y: Proximal persistent $\mathrm{Na}^{+}$channels drive spike afterdepolarizations and associated bursting in adult CA1 pyramidal cells. J Neurosci 2005;25:9704-9720.

42 Jahnsen H, Llinas R: Ionic basis for the electro-responsiveness and oscillatory properties of guinea-pig thalamic neurones in vitro. J Physiol 1984;349:227-247.

-43 Wu N, Enomoto A, Tanaka S, Hsiao CF, Nykamp DQ, Izhikevich E, Chandler SH: Persistent sodium currents in mesencephalic $\mathrm{V}$ neurons participate in burst generation and control of membrane excitability. J Neurophysiol 2005;93:2710-2722.

44 Cepeda C, Chandler SH, Shumate LW, Levine MS: Persistent $\mathrm{Na}^{+}$conductance in medium-sized neostriatal neurons: characterization using infrared videomicroscopy and whole cell patch-clamp recordings. J Neurophysiol 1995; 74:1343-1348.

-45 Baker MD, Bostock H: Low-threshold, persistent sodium current in rat large dorsal root ganglion neurons in culture. J Neurophysiol 1997;77:1503-1513.

46 Magistretti J, Alonso A: Biophysical properties and slow voltage-dependent inactivation of a sustained sodium current in entorhinal cortex layer-II principal neurons: a whole-cell and single-channel study. J Gen Physiol 1999;114:491-509.

47 Manis PB, Molitor SC, Wu H: Subthreshold oscillations generated by TTX-sensitive sodium currents in dorsal cochlear nucleus pyramidal cells. Exp Brain Res 2003;153:443-451.

-48 Boehmer G, Greffrath W, Martin E, Hermann S: Subthreshold oscillation of the membrane potential in magnocellular neurones of the rat supraoptic nucleus. J Physiol 2000;526:115-128.

49 Agrawal N, Hamam BN, Magistretti J, Alonso A, Ragsdale DS: Persistent sodium channel activity mediates subthreshold membrane potential oscillations and low-threshold spikes in rat entorhinal cortex layer $\mathrm{V}$ neurons. Neuroscience 2001;102:53-64.

50 Reboreda A, Sanchez E, Romero M, Lamas JA: Intrinsic spontaneous activity and subthreshold oscillations in neurones of the rat dorsal column nuclei in culture. J Physiol 2003;551:191-205.

51 Lee RH, Heckman CJ: Bistability in spinal motoneurons in vivo: systematic variations in rhythmic firing patterns. J Neurophysiol 1998;80:572-582. 
52 Powers RK, Binder MD: Persistent sodium and calcium currents in rat hypoglossal motoneurons. J Neurophysiol 2003;89:615-624.

53 Schwindt PC, Crill WE: Amplification of synaptic current by persistent sodium conductance in apical dendrite of neocortical neurons. J Neurophysiol 1995;74:2220-2224.

54 Lipowsky R, Gillessen T, Alzheimer C: Dendritic $\mathrm{Na}^{+}$channels amplify EPSPs in hippocampal CA1 pyramidal cells. J Neurophysiol 1996;76:2181-2191.

55 Lee RH, Heckman CJ: Adjustable amplification of synaptic input in the dendrites of spinal motoneurons in vivo. J Neurosci 2000;20:6734-6740.

56 Kang Y, Saito M, Sato H, Toyoda H, Maeda Y, Hiral T, Bae YC: Involvement of persistent $\mathrm{Na}^{+}$current in spike initiation in primary sensory neurons of the rat mesencephalic trigeminal nucleus. J Neurophysiol 2007;97: 2385-2393.

57 Enomoto A, Han JM, Hsiao CF, Wu N, Chandler SH: Participation of sodium currents in burst generation and control of membrane excitability in mesencephalic trigeminal neurons. J Neurosci 2006;26:3412-3422.

-58 Enomoto A, Han JM, Hsiao CF, Chandler SH: Sodium currents in mesencephalic trigeminal neurons from $\mathrm{Na}_{\mathrm{v}} 1.6$ null mice. J Neurophysiol 2007;98:710-719.

59 Tanaka S, Chandler SH: Serotonergic modulation of persistent sodium currents and membrane excitability via cyclic AMP-protein kinase A cascade in mesencephalic V neurons. J Neurosci Res 2006;83:1362-1372.

60 Santoro B, Chen S, Luthi A, Pavlidis P, Shumyatsky GP, et al: Molecular and functional heterogeneity of hyperpolarization-activated pacemaker channels in the mouse CNS. J Neurosci 2000;20:5264-5275.

61 Ludwig A, Zong X, Jeglitsch M, Hofmann F, Biel M: A family of hyperpolarization-activated mammalian cation channels. Nature 1998;393:587-591.

62 Halliwell JV, Adams PR: Voltage-clamp analysis of muscarinic excitation in hippocampal neurons. Brain Res 1982;250:71-92.

63 Brown DA, Gahwiler BH, Griffith WH, Halliwell JV: Membrane currents in hippocampal neurons. Prog Brain Res 1990;83:141-160.

64 Robinson RB, Siegelbaum SA: Hyperpolarization-activated cation currents: from molecules to physiological function. Annu Rev Physiol 2003;65:453-480.

65 Khakh BS, Henderson G: Hyperpolarization-activated cationic currents $\left(I_{h}\right)$ in neurones of the trigeminal mesencephalic nucleus of the rat. J Physiol 1998;510:695-704.

66 Pape HC: Queer current and pacemaker: the hyperpolarization-activated cation current in neurons. Annu Rev Physiol 1996;58:299-327.

-67 Ghamari-Langroudi M, Bourque CW: Excitatory role of the hyperpolarization-activated inward current in phasic and tonic firing of rat supraoptic neurons. J Neurosci 2000;20:4855-4863.

68 Lupica CR, Bell JA, Hoffman AF, Watson PL: Contribution of the hyperpolarization-activated current $\left(I_{h}\right)$ to membrane potential and GABA release in hippocampal interneurons. J Neurophysiol 2001;86:261-268.

69 Huang W, Xiu Y, Yan JA, He WJ, Zhao YD, Hu ZA, Ruan HZ: Facilitation of $I_{\mathrm{h}}$ channels by P2Y ${ }_{1}$ receptors activation in mesencephalic trigeminal neurons. Neurosci Lett 2010;482:156-159.

-70 Kang Y, Notomi T, Saito M, Zhang W, Shigemoto R: Bidirectional interactions between h-channels and $\mathrm{Na}^{+}-\mathrm{K}^{+}$ pumps in mesencephalic trigeminal neurons. J Neurosci 2004;24:3694-3702.

71 Min MY, Hsu PC, Yang HW: The physiological and morphological characteristics of interneurons caudal to the trigeminal motor nucleus in rats. Eur J Neurosci 2003;18:2981-2998.

72 Liu Y, Yang J, Hu S: Transition between two excitabilities in mesencephalic V neurons. J Comput Neurosci 2008; 24:95-104.

73 Gong Y, Xu J, Ren W, Hu S, Wang F: Determining the degree of chaos from analysis of ISI time series in the nervous system: a comparison between correlation dimension and nonlinear forecasting methods. Biol Cybern 1998;78:159-165.

74 Ren XS: Marital status and quality of relationships: the impact on health perception. Soc Sci Med 1997;44: 241-249.

75 Hsiao CF, Kaur G, Vong A, Bawa H, Chandler SH: Participation of $\mathrm{K}_{\mathrm{v}} 1$ channels in control of membrane excitability and burst generation in mesencephalic V neurons. J Neurophysiol 2009;101:1407-1418.

76 Amir R, Michaelis M, Devor M: Membrane potential oscillations in dorsal root ganglion neurons: role in normal electrogenesis and neuropathic pain. J Neurosci 1999;19:8589-8596.

77 Wilcox KS, Gutnick MJ, Christoph GR: Electrophysiological properties of neurons in the lateral habenula nucleus: an in vitro study. J Neurophysiol 1988;59:212-225.

-78 Silva LR, Amitai Y, Connors BW: Intrinsic oscillations of neocortex generated by layer 5 pyramidal neurons. Science 1991;251:432-435.

79 Klink R, Alonso A: Ionic mechanisms for the subthreshold oscillations and differential electroresponsiveness of medial entorhinal cortex layer II neurons. J Neurophysiol 1993;70:144-157.

80 Amitai Y: Membrane potential oscillations underlying firing patterns in neocortical neurons. Neuroscience 1994;63:151-161.

81 Bal T, McCormick DA: Synchronized oscillations in the inferior olive are controlled by the hyperpolarizationactivated cation current $I_{\mathrm{h}}$. J Neurophysiol 1997;77:3145-3156.

82 Hutcheon B, Miura RM, Puil E: Models of subthreshold membrane resonance in neocortical neurons. J Neurophysiol 1996;76:698-714 
83 Llinas R, Yarom Y: Oscillatory properties of guinea-pig inferior olivary neurones and their pharmacological modulation: an in vitro study. J Physiol 1986;376:163-182.

84 Pelkey KA, Marshall KC: Actions of excitatory amino acids on mesencephalic trigeminal neurons. Can J Physiol Pharmacol 1998;76:900-908.

-85 Xing JL, Hu SJ, Long KP: Subthreshold membrane potential oscillations of type A neurons in injured DRG. Brain Res 2001;901:128-136.

86 Song Y, Li HM, Xie RG, Yue ZF, Song XJ, Hu SJ, Xing JL: Evoked bursting in injured A $\beta$ dorsal root ganglion neurons: a mechanism underlying tactile allodynia. Pain 2012;153:657-665.

87 Inoue T, Watanabe S, Kawahara S, Kirino Y: Phase-dependent filtering of sensory information in the oscillatory olfactory center of a terrestrial mollusk. J Neurophysiol 2000;84:1112-1115.

88 Collins JJ, Imhoff TT, Grigg P: Noise-enhanced tactile sensation. Nature 1996;383:770.

89 Russell DF, Wilkens LA, Moss F: Use of behavioural stochastic resonance by paddle fish for feeding. Nature 1999;402:291-294.

-90 Freund JA, Schimansky-Geier L, Beisner B, Neiman A, Russell DF, Yakusheva T, Moss F: Behavioral stochastic resonance: how the noise from a Daphnia swarm enhances individual prey capture by juvenile paddlefish. J Theor Biol 2002;214:71-83.

91 Moss F, Ward LM, Sannita WG: Stochastic resonance and sensory information processing: a tutorial and review of application. Clin Neurophysiol 2004;115:267-281.

-92 Baker R, Llinas R: Electrotonic coupling between neurones in the rat mesencephalic nucleus. J Physiol 1971; 212:45-63.

93 Liem RS, Copray JC, van Willigen JD: Ultrastructure of the rat mesencephalic trigeminal nucleus. Acta Anat (Basel) 1991;140:112-119.

94 Luo P, Dessem D: Morphological evidence for recurrent jaw-muscle spindle afferent feedback within the mesencephalic trigeminal nucleus. Brain Res 1996;710:260-264.

95 Curti S, Hoge G, Nagy JI, Pereda AE: Synergy between electrical coupling and membrane properties promotes strong synchronization of neurons of the mesencephalic trigeminal nucleus. J Neurosci 2012;32:4341-4359.

$\$ 96$ Curti S, Hoge G, Nagy JI, Pereda AE: Electrical transmission between mammalian neurons is supported by a small fraction of gap junction channels. J Membr Biol 2012;245:283-290.

-97 Park H, Yamada K, Kojo A, Sato S, Onozuka M, Yamamoto T: Drebrin (developmentally regulated brain protein) is associated with axo-somatic synapses and neuronal gap junctions in rat mesencephalic trigeminal nucleus. Neurosci Lett 2009;461:95-99.

\$8 Davoine F, Curti S, Monzon P: Modeling of sodium currents from mesencephalic trigeminal neurons by system identification and sensitivity analysis. Neuroscience 2013;14(suppl 1):P75. 